\title{
Warning: Attending to a mask may be hazardous to your perception
}

\author{
MATTHEW S. TATA and DEBORAH E. GIASCHI \\ University of British Columbia, Vancouver, British Columbia, Canada
}

\begin{abstract}
Object substitution is a type of backward masking that occurs when a mask appears during visual search for a target. We tested the hypothesis that object substitution is an overwriting process triggered by attentional selection of the mask. Impeding attentional selection of a mask by embedding it in an array of distractors eliminated object substitution. Similarly, object substitution did not occur when the mask appeared in advance of the target and, therefore, could not capture attention during search for the target. However, masking was reinstated when the mask was revealed from background contours at the moment of target onset and could therefore capture attention during search. These observations demonstrate that attentional selection of the mask is a necessary step in this type of masking and suggest that object substitution is active overwriting of unattended information triggered by selection of other visual information at a nearby location.
\end{abstract}

Masking is a reduction in the visibility of a visual target due to the presence of another visual stimulus (the mask) at or near the same location (see Breitmeyer, 1984). Most investigations of masking have focused on spatial and temporal relationships, such as the spacing between target and mask contours and the onset asynchrony of the stimuli (SOA). More recently, it has become clear that the complexity of the display in which a target appears affects the strength of masking in a variety of situations: metacontrast masking, in which a non-overlapping target and mask are presented in sequence (Averbach \& Coriell, 1961; Enns \& Di Lollo, 1997; Ramachandran \& Cobb, 1995; Shelley-Tremblay \& Mack, 1999; Tata, 2002; Uttal, 1970); pattern masking, in which the mask contours physically overlap those of the target (Spencer \& Shuntich, 1970); and common-onset masking, in which the mask appears at the same time as the target but outlives it by a variable duration (Di Lollo, Enns, \& Rensink, 2000; Neill, Hutchison, \& Graves, 2002).

The influence of display complexity on the strength of masking has been interpreted in terms of selective attention (Breitmeyer \& Ganz, 1976; Di Lollo et al., 2000). In this view, an unattended target is encoded by the visual system in an impoverished or nonpermanent state and is thus particularly vulnerable to the effect of a subsequent masking stimulus. The term object substitution (Enns \& Di Lollo, 1997, 2000) is now commonly used to describe

Funding for this research was provided by NSERC Canada Grant OGPO194526 to D.G. The authors thank Vince Di Lollo, Robert Dougherty, Lawrence Ward, and W. Trammell Neill for their useful comments on earlier drafts of the manuscript. Correspondence concerning this article should be addressed to M. S. Tata, Department of Psychology and Neuroscience, Canadian Centre for Behavioural Neuroscience, University of Lethbridge, 4401 University Dr., Lethbridge, AB, T1K 3M4 Canada (e-mail: matthew.tata@uleth.ca). the particularly strong backward masking that arises when attentional selection of the target is impaired or delayed. Although the theoretical implications of this term remain unconfirmed, it is thought that the impoverished representation of the target is overwritten by the subsequent representation of the mask (see Tata, 2002, for a discussion of the merits of various terms used to describe masking phenomena).

Selectively attending to the target modulates backward masking, but what about selectively attending to the mask? Shelley-Tremblay and Mack (1999) showed that highly salient stimuli acted as more powerful masks relative to masks of lower salience. They concluded that attention focused on the mask increased the effectiveness of that mask. Tata and Giaschi (1999) and Tata (2002) hypothesized that object substitution is not simply modulated by attention to the mask, but is dependent on it. In their theory, attentional selection of the mask during search for the target triggers a mechanism that suppresses unattended information at that location and overwrites it with a representation of the attended mask.

The possibility that attentional selection of the mask plays a role in object substitution has been addressed in a series of experiments in which Neill et al. (2002) manipulated attentional factors related to the mask. This reduced the likelihood that the observer would focus attention on the mask. In their Experiment 2, Neill et al. used a central arrow to indicate the target location and presented masks around both the target and the distractor so that subjects would not actively use the mask as a cue to the target location. Masking in this situation was reduced relative to that of a single informative mask, but it was still robust. They concluded that voluntary attention directed to the mask modulates the effect but is not necessary to generate object substitution. Their Experiment 3 showed that a single randomly placed mask (which would 
presumably not be voluntarily attended) generated strong object substitution when it occurred at the target location, but impaired target identification weakly when it occurred at the nontarget location. Taken together, these results suggest that a reflexive capture of attention by the mask at the target location is a critical component of object substitution.

Neill et al. (2002) also reported an intriguing variation in mask effectiveness across mask onset times: A mask appearing as little as $133 \mathrm{msec}$ in advance of the target was ineffective, whereas other studies (Enns \& Di Lollo, 1997; Tata, 2002) have shown that a mask appearing after the target at about the same SOA produced very strong object substitution. Neill et al. observed that "it is unclear why one onset asymmetry would protect the target from masking but the opposite asymmetry would not" (p. 693).

In the present study, we examine the hypothesis that object substitution involves an attentional selection of the mask and explore the explanatory power of this hypothesis. For example, the onsets of items in a display are known to affect the sequence in which attention is deployed to them: New perceptual objects tend to capture attention (see, e.g., Yantis \& Jonides, 1984), and new items in a display are afforded higher priority than older items (Yantis \& Johnson, 1990). Thus, the asymmetrical effect of mask onset time noted by Neill et al. (2002) may be explained if masking by object substitution occurs when the masking object captures attention. As was suggested by the work of Yantis and colleagues, this attentional capture will not occur if the mask precedes the target, but it could occur if it appears at the same moment as the target or later.

If object substitution occurs because the mask at the target location captures attention, then preventing capture by the mask should eliminate object substitution. In Experiment 1 of the present study, we tested whether an increase in the set size of masks (to eight) would reduce or eliminate object substitution. In Experiments 2 and 3, we tested the assumption that the onset of the mask as a new perceptual object critically modulates its effectiveness. Experiment 2 shows that an "old" mask is incapable of masking a "new" target, whereas Experiment 3 shows that converting "old" contours into a "new" mask at the moment of target onset reinstates masking.

\section{EXPERIMENT 1}

Manipulation of set size is a technique commonly used to prevent immediate focusing of attention on a single stimulus (see, e.g., Treisman \& Gelade, 1980). Displays that generate object substitution include distractors to prevent rapid selection of the target. However, in such displays, the target and distractors vanish, leaving a single mask without other stimuli that might compete for attention. We hypothesized that this situation allows for rapid attentional selection of the mask, which thereby triggers object substitution. We predicted that increasing the set size of masks to prevent immediate attentional selection of the particular mask at the target location would eliminate object substitution.

\section{Method}

In the present experiments, we chose to employ a variation of the common-onset paradigm used by Di Lollo et al. (2000). In this paradigm, both the target and the mask appear simultaneously (with an SOA of zero), but the target vanishes within a few tens of milliseconds, leaving the mask alone for a variable mask duration. Nine observers viewed two consecutive frames of stimuli presented on a computer monitor, from a distance of $57 \mathrm{~cm}$. Frame 1 contained the items of a visual search task uniformly distributed around a notional circle of $3^{\circ}$ radius centered at fixation. The target of the search was a single complete ring (an $\mathrm{O}$ ) located randomly among seven distractor rings with gaps (Cs; see Figure 1). On $50 \%$ of trials, no target was present and Frame 1 contained only eight Cs. The observer's task was to indicate whether the target was present or absent in this display. Target and distractors subtended $1^{\circ}$ of visual angle, and the gap in the distractors was $90^{\circ}$ of arc. Also appearing in Frame 1 were eight squares that served as common-onset masks, each surrounding an item of the search array. The squares were $2^{\circ}$ in width for all but 1 observer, who required a slightly larger targetmask spacing to obtain above-threshold visibility of the target.

Frame 1 was presented for $10 \mathrm{msec}$ and was followed immediately by Frame 2 . Frame 2 contained either the single mask that had surrounded the target in Frame 1 or all eight masks from Frame 1. In the single-mask condition on target-absent trials, a mask remained visible at the location of one of the distractors. The mask duration was $0,40,80,160,320,500$, or $750 \mathrm{msec}$, with duration $=$ 0 indicating that the target and the masks vanished together. All the observers completed 30 trials in each of the 2 (set sizes) $\times 7$ (mask durations) conditions. Perceived brightness of the masks was matched across the entire range of mask durations, through a method-ofadjustment procedure similar to that described by Di Lollo et al. (2000).

\section{Results}

Sensitivity to detect the presence of the target (expressed as $d^{\prime}$ ) was averaged across observers as a function of mask duration and is presented in Figure 2. Sensitivity for the two set-size conditions was nearly identical at a mask duration of $0 \mathrm{msec}$, because Frame 2 in both conditions was blank and these stimuli were identical. However, sensitivity in the two set-size conditions at a 40-msec mask duration differed markedly and followed different masking functions with increasing mask duration. This interaction between set size and mask duration was shown to be significant in a 2 (set-size condition) $\times 7$ (mask duration) repeated measures analysis of variance [ANOVA; $F(6,48)=17.538, p<.001$ ]. Sensitivity to detect the target in the single-mask condition was maximal at the short mask duration of $40 \mathrm{msec}$ and then decreased with increasing mask duration $[F(6,48)=$ $29.468, p<.001$ ], approaching a $d^{\prime}$ of zero (chance performance) at a mask duration of $750 \mathrm{msec}$. By contrast, a small drop in sensitivity was observed with a mask set size of eight between 0 and $40 \mathrm{msec}$, but no significant overall effect of mask duration was observed in the eightmask condition $[F(6,48)=1.57, p=.17]$. 


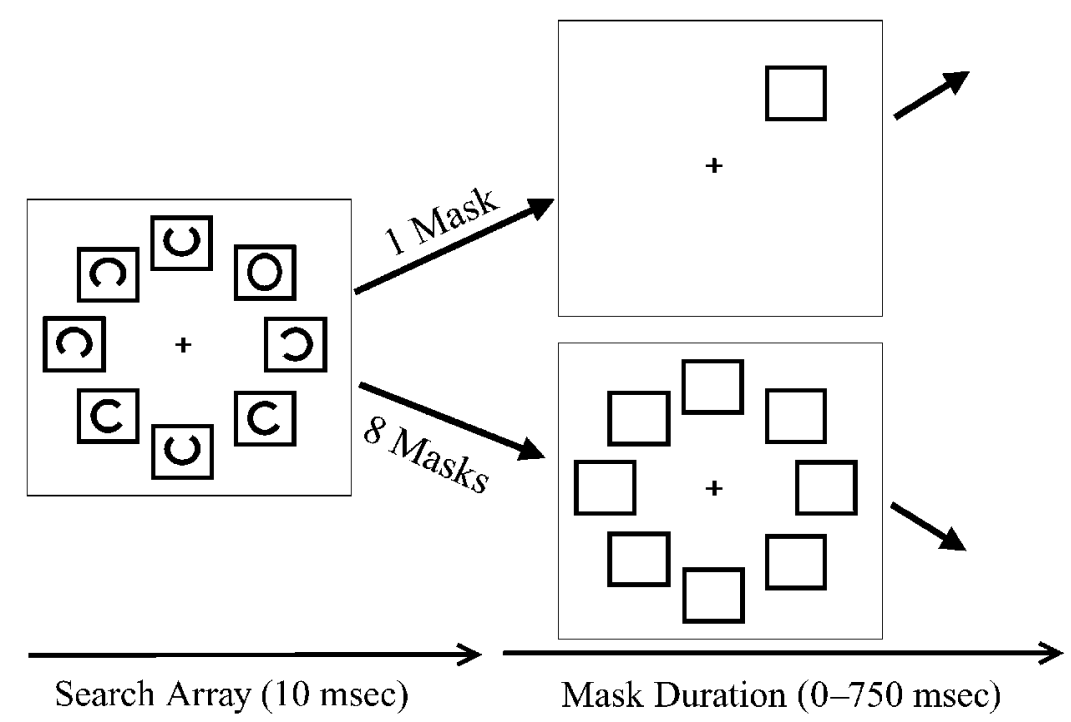

Figure 1. Schematic of the stimuli used in Experiment 1. Frame 1 (left) contained a search array with each element of the search appearing inside a square (commononset masking). The observer's task was to detect the presence of an $\mathrm{O}$ among Cs. On catch trials, the array contained only Cs. The search array vanished after $10 \mathrm{msec}$, leaving either the single square at the target location (top right) or all eight squares (bottom right). Frame 2 remained visible for a duration of $0-750 \mathrm{msec}$.

\section{Discussion}

The masking functions obtained in Experiment 1 differ somewhat from those of previous studies (e.g., Di Lollo et al., 2000) and call for careful interpretation. In particular, whereas in previous studies maximal target visibility was reported when the target and mask offset simultaneously, target visibility in the single-mask condition of the present study was maximal with a slight offset asynchrony (mask duration of $40 \mathrm{msec}$ ). Visibility then dropped from a maximum $d^{\prime}$ of 2.72 at $40 \mathrm{msec}$ to around chance performance at $750 \mathrm{msec}$. This spike in visibility at the first nonzero mask duration probably reflects the use of the mask as a postcue that indicated the location of the target. Unlike in previous studies, this was impossible with a 0 -msec mask duration, since each location had an identical mask (Figure 1). Nevertheless, the steady decline in visibility of the target with increasing nonzero mask durations can be attributed to strong object substitution masking. The situation was different for the eightmask condition. Following an initial drop of about 0.75 $d^{\prime}$ units between the 0 -msec and the 40 -msec mask durations, sensitivity to detect the target remained constant at above threshold $\left(d^{\prime}=1.0\right)$ across all mask durations. This overall decrement in visibility with nonzero mask durations might be due to low-level lateral masking of the target contours by the contours of the mask, but it is inconsistent with object substitution because it did not increase in magnitude with increasing mask duration.

These results are consistent with the hypothesis that object substitution depends on attentional selection of the mask. The absence of object substitution in the eight- mask condition suggests that selection of the mask is necessary for object substitution to occur. It is possible, however, that object substitution is simply made stronger when attention is focused on the mask and that a ceiling effect in the eight-mask condition made it impossible to observe a reduction in visibility with increasing mask duration.

It is clear that focusing attention on the mask plays some role in object substitution. Whether this involves voluntary or involuntary attention is less clear. If the observers were actively attending to the mask to use it as a

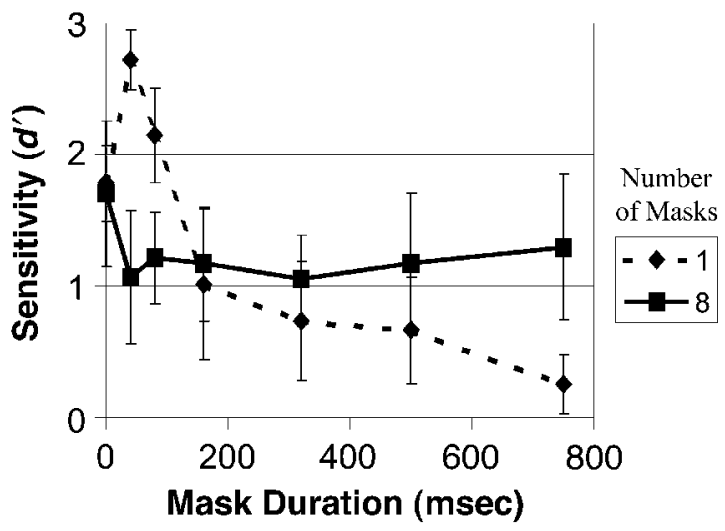

Figure 2. Experiment 1: sensitivity $\left(d^{\prime}\right)$ to detect the presence of the target as a function of mask duration for the single-mask and eight-mask conditions, averaged across 9 observers. Error bars represent the $95 \%$ confidence interval. 
cue to the target location, it is unclear why voluntarily attending to a 40-msec mask should aid perception of the target whereas attending to a 160 -msec mask should hinder it. What happens during that extra $120 \mathrm{msec}$ ? One argument might be that the process of object substitution occurs over time. Perhaps effective masking requires many tens of milliseconds during which only the mask is visible. This suggestion is untenable, however, because Tata (2002) showed that strong object substitution can be obtained with targets and masks each lasting only $10 \mathrm{msec}$, provided that the mask appears about 100 to $300 \mathrm{msec}$ after the target. Alternatively, the spike in sensitivity at $40 \mathrm{msec}$ might occur because the temporal discontinuity associated with a briefly lingering mask led to an involuntary rather than a voluntary shift of attention to an integrated percept of the target and mask. We are led to the hypothesis that object substitution depends more critically on involuntary attentional capture by the mask than on a voluntary selection of it. Experiments 2 and 3 address this second possibility.

\section{EXPERIMENT 2}

In Experiment 1, we manipulated mask set size to show that attentional selection of the mask might be involved in object substitution. Other attentional manipulations of the mask might also modulate object substitution. Of particular interest is that abrupt onsets of visual objects capture attention under certain conditions (Yantis \& Jonides, 1984) and that the visual system assigns priority tags to objects in a scene, with attention being deployed to new items in a display before older items (Yantis \& Johnson, 1990). If masking occurs when attention is captured by the mask during search for the target, then a mask appearing several hundred milliseconds before the target and distractors should be ineffective, because attention will be deployed immediately and solely to the items in the search array when they appear.

\section{Method}

The method of Experiment 2 was similar to that of the singlemask condition of Experiment 1, except for the insertion of a preview frame (Frame 1) in advance of the search array (now Frame 2). Frame 1 was presented for $200 \mathrm{msec}$, and its contents depended on the experimental condition (Figure 3 ). In the no-preview condition, Frame 1 was blank and the masks, target, and distractors appeared simultaneously at the onset of Frame 2 . In the preview condition, Frame 1 contained a square mask at each possible target location (to prevent spatial precuing of attention), and the target and distractors appeared within these squares after $200 \mathrm{msec}$. Because the displays used in the present experiment were designed to be used in Experiment 3 as well, the eccentricities of the items in the array varied slightly. Items at the 12:00, 3:00, 6:00, and 9:00 positions were located at $4.5^{\circ}$ eccentricity, whereas the remaining four items were at $4.14^{\circ}$ eccentricity. Four observers were tested in four 16-trial blocks for each of the 2 (preview conditions) $\times 5$ (mask durations) conditions.

\section{Results}

Sensitivity to detect the target averaged across 4 observers appears as a function of mask duration in Figure 4. The pattern of masking in the no-preview condition is similar to that seen in the single-mask condition of Ex-

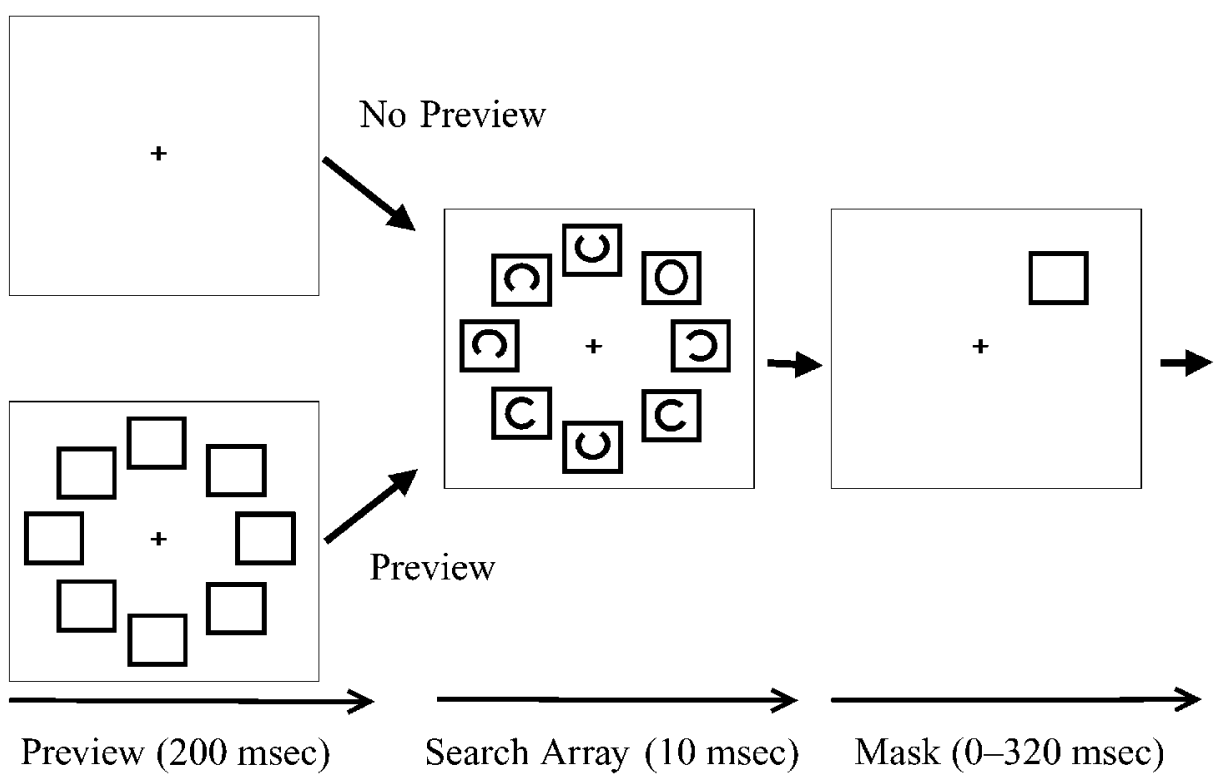

Figure 3. Schematic of stimulus used in Experiment 2. Each trial began with either a blank screen (no-preview condition) or eight square masks (preview condition) at all possible subsequent target locations. This 200-msec preview was followed by the onset of an element of the search task within each square. The observer's task was to detect the presence of an $\mathrm{O}$ among $\mathrm{Cs}$, with catch trials having only Cs present. The search array vanished after $10 \mathrm{msec}$, leaving a single square at the target location to act as a mask. 


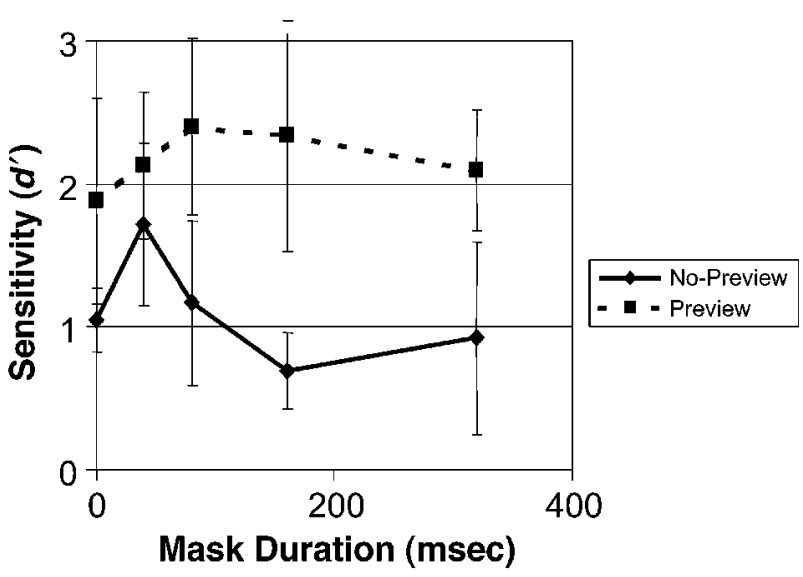

Figure 4. Experiment 2: sensitivity $\left(d^{\prime}\right)$ to detect the presence of the target as a function of mask duration for preview and nopreview conditions, averaged across 4 observers. Error bars represent the $95 \%$ confidence interval.

periment 1 . Sensitivity peaked at a mask duration of $40 \mathrm{msec}$ and then began to decrease asymptotically with increasing mask duration. The pattern observed for the preview condition was quite different. Sensitivity at a mask duration of zero was greater than sensitivity in the no-preview condition. However, unlike in the no-preview condition, this high degree of sensitivity did not drop off with increasing mask duration. The interaction in the 2 (preview) $\times 5$ (mask duration) repeated measures ANOVA was significant $[F(4,12)=4.39, p<.05]$.

\section{Discussion}

Object substitution did not occur when the mask was previewed prior to the onset of the search array. However, as in Experiment 1, strong object substitution was evidenced by the asymptotic decrease in sensitivity to detect the target beginning at the first nonzero mask duration. Importantly, since identical masks appeared at every possible target location, the absence of masking in the preview condition was not because attention was cued to the target location in advance of target onset, as described by Di Lollo et al. (2000). This absence must instead be explained in terms of the relative timing of the onsets of the various elements in the display. Since the mask appeared before the search array, it could not capture attention during search for the target. Sustained presence of a mask at the target location was not itself sufficient to generate object substitution. It is the temporal order in which the visual system attempts to deal with the objects in the display that determines whether or not object substitution will occur. The situation might be conceptualized as a competition for selection between the target and the mask. Previewing the mask biased this competition in favor of the target, since it was the "new" object at that location.

The results of Experiment 2 also speak to the possibility that the observers tended to use the mask as a cue to the target location. Notice that from the moment of target onset, the single-mask condition in Experiment 1 and both conditions in Experiment 2 involved an identical display sequence. The mask was equally informative in each of these conditions. Nevertheless, no object substitution occurred in the preview condition of Experiment 2. Factors other than the information content of the mask modulated object substitution. We are left with the conclusion that it was involuntary rather than voluntary selection of the mask that played a role in the masking effect.

\section{EXPERIMENT 3}

Experiment 2 showed that object substitution does not occur when the mask cannot capture attention during search for the target. We also predicted that enabling attentional capture by the mask would reinstate masking, even if the mask's contours had been previewed. In Experiment 3, we introduced a background grid during the preview period, which hid the mask contours (Figure 5). Thus, by revealing the mask from the background grid, we caused it to appear as a new object at the same time as the appearance of the search array, despite the fact that its contours had been previewed for $200 \mathrm{msec}$, as in Experiment 2 . The release from masking conferred by preregistering the mask as a visual object should be eliminated in this case.

\section{Method}

Three of the 4 observers tested in Experiment 2 also participated in Experiment 3. The procedure was identical to that of Experiment 2 except that the square masks in Frames 1 and 2 were replaced by a grid covering the entire screen (Figure 5). The contours of the grid were constructed in such a way that the contours of the mask appearing in Frame 3 were also present in the grid. In effect, the mask was hidden in the background of the display. This allowed the contours constituting the mask to appear $200 \mathrm{msec}$ prior to onset of the search array, whereas the mask itself was not revealed as a distinct object until $210 \mathrm{msec}$ later, with the onset of Frame 3.

\section{Results}

Mean sensitivity for the 3 observers is plotted as a function of mask duration in Figure 6. This is superimposed on the results from Experiment 2 for the same 3 observers. Sensitivity at a mask duration of zero was greater in the grid condition than in either the preview or the nopreview condition. However, the pattern that emerged as mask duration increased was the same as those of the single-mask condition in Experiment 1 and the no-preview condition in Experiment 2. Sensitivity to detect the target dropped asymptotically from a maximum at the mask duration of $40 \mathrm{msec}$. A 3 (preview condition) $\times 5$ (mask duration) repeated measures ANOVA was performed for the 3 observers who participated in both Experiments 2 and 3 . The interaction between preview condition and mask duration was significant $[F(8,16)=5.814, p=$ $.001]$. 


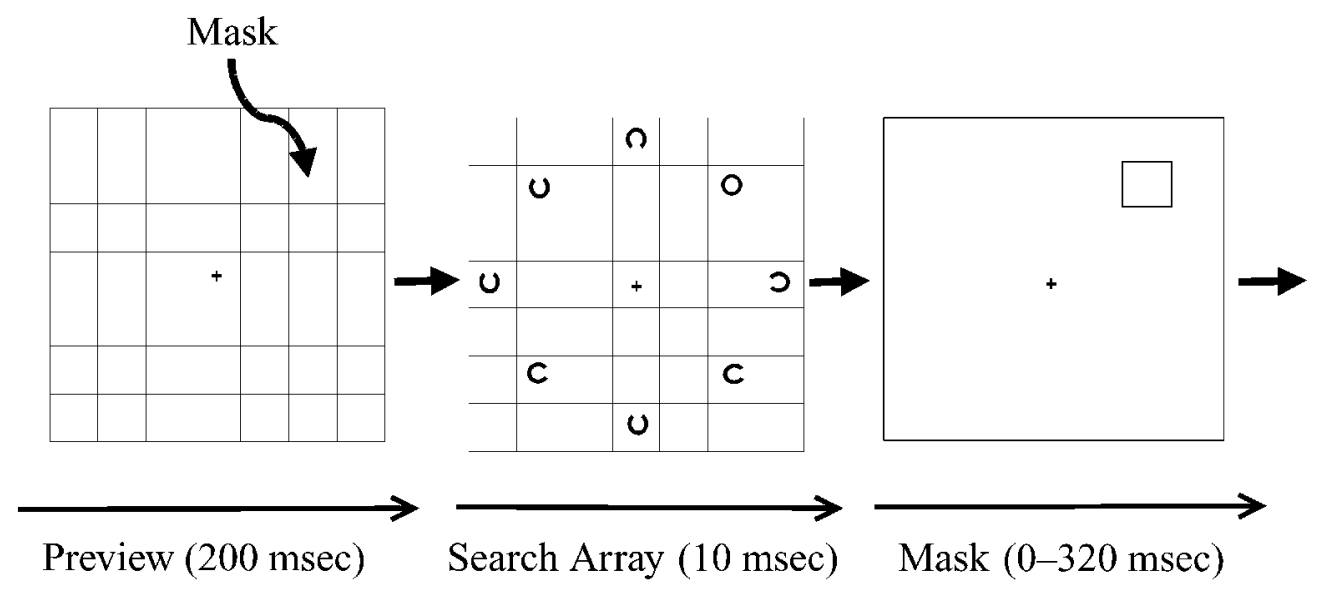

Figure 5. Schematic of stimulus used in Experiment 3. The preview of square masks in Experiment 2 was replaced by a full-screen grid. The lines of the grid were such that each square in the grid was identical to the square used as the mask in the previous experiments. After a 200 -msec preview, a search array appeared embedded in the grid. The observer's task was to detect the presence of an $\mathrm{O}$ among Cs. After $10 \mathrm{msec}$, the search array as well as the grid vanished, leaving only the contours of a square mask at the target location. Notice that these contours, which define the mask in the final frame, remained unchanged throughout the entire trial.

\section{Discussion}

As we predicted, hiding the mask in a background grid negated any advantage gained by previewing it for $200 \mathrm{msec}$, despite the fact that the contours of the mask were visible during the preview period. Thus, it is the appearance of new visual objects during search for the target that influences object substitution. This observation lends some validity to the term object substitution itself. Indeed, this is a phenomenon involving the interaction of object representations rather than simply the constituent features of objects.

\section{CONCLUSION}

The context, both spatial and temporal, in which a mask appears has been shown to modulate object substi-

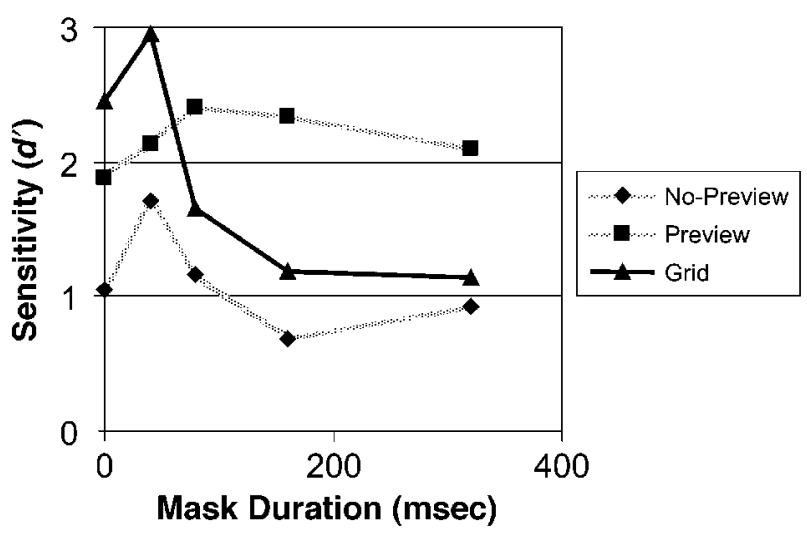

Figure 6. Experiment 3: sensitivity $\left(d^{\prime}\right)$ to detect the target as a function of mask duration, averaged across observers. The results of the grid preview condition are superimposed on the results from the preview and no-preview conditions of Experiment 2. tution. As in previous studies of object substitution, the manipulation of attentional factors (in this case, set size and the abrupt onsets of visual objects) determined whether or not object substitution occurred. In this study, however, these manipulations pertained exclusively to the mask.

Di Lollo et al. (2000) have advanced a theory to explain the processes by which a target may be either masked or successfully perceived. They suggested that object substitution arises as a result of a mismatch between visual representations of the previous target and of the subsequent mask. Other investigators (Neill et al., 2002; Shelley-Tremblay \& Mack, 1999) have demonstrated that the focusing of attention on the mask modulates the strength of object substitution. Here, we propose to extend the theory of Di Lollo et al. by including an active role for attentional selection of the mask. We base this proposal on three observations. First, Tata (2002) found that object substitution does not occur if sufficient time is allowed to complete visual search for the target. Second, in the present study we found that a simple mismatch between an initial target and a subsequent mask, which should yield object substitution according to Di Lollo et al.'s theory, was insufficient to produce the effect. Finally, we found that object substitution occurred only when attentional selection of the mask was encouraged, as in the single-mask condition of Experiment 1 and the no-preview condition of Experiment 2, but not when it was discouraged, as in the eight-mask condition of Experiment 1 and the preview condition of Experiment 2 . We suggest, therefore, that attentional selection of the mask during search for the target leads to object substitution-a notion that is not incompatible with Di Lollo et al.'s model. As in their model, object substitution might be thought to involve a competition for selec- 
tion between target and mask. Factors that bias this selection process toward the mask and away from the target in the critical tens of milliseconds after the target has vanished from the screen, but before it has been attended, will increase the likelihood of object substitution.

Although it seems likely that attention must be focused on the mask for object substitution to occur, it is less clear whether it matters how attention is deployed. The results reported by Neill et al. (2002) suggest that involuntarily attending to the mask is sufficient to generate object substitution. The present study supports that conclusion. Experiments 2 and 3 demonstrated that an informative mask produced strong object substitution only if it was capable of involuntarily capturing attention. It may be that voluntary selection of the mask is also sufficient to generate object substitution in some situations; however, this has not been explicitly tested.

The importance of selective attention in determining what is perceived and what is not perceived has been demonstrated in a variety of ways, such as in terms of inattentional blindness (Mack \& Rock, 1998), change blindness (Rensink, O’Regan, \& Clark, 1997; Simons \& Levin, 1998), and the attentional blink (Raymond, Shapiro, \& Arnell, 1992). In these situations, it is typically the absence of focused attention that leads to failures of perception. In the present study, this notion is carried further: The focusing of attention on one aspect of a visual scene can dramatically affect the representation of other (nearby) parts of that scene. This observation emphasizes that selective attention is an active process, not simply a necessary precondition for visual perception.

\section{REFERENCES}

AVERBACH, E., \& CORIELl, A. S. (1961). Short-term memory in vision. Bell System Technical Journal, 40, 309-328.

BREITME YER, B. G. (1984). Visual masking: An integrative approach. New York: Oxford University Press.

BREITMEYER, B. G., \& GANZ, L. (1976). Implications of sustained and transient channels for theories of visual pattern masking, saccadic suppression, and information processing. Psychological Review, 83, $1-36$.

Di Lollo, V., EnNS, J. T., \& Rensink, R. A. (2000). Competition for consciousness among visual events: Psychophysics of reentrant pathways. Journal of Experimental Psychology: General, 129, 481-507.

ENNS, J. T., \& Di Lollo, V. (1997). Object substitution: A new form of masking in unattended visual locations. Psychological Science, $\mathbf{8}$, 135-139.

EnNS, J. T., \& Di Lollo, V. (2000). What's new in visual masking? Trends in Cognitive Sciences, 4, 345-352.

MACK, A., \& ROCK, I. (1998). Inattentionalblindness. Cambridge, MA: MIT Press.

Neill, W. T., Hutchison, K. A., \& Graves, D. F. (2002). Masking by object substitution: Dissociation of masking and cuing effects. Journal of Experimental Psychology: Human Perception \& Performance, 28, 682-694.

RAMACHANDRAN, V. I., \& COBB, S. (1995). Visual attention modulates metacontrast masking. Nature, 373, 66-68.

RAYMOnd, J. E., Shapiro, K. L., \& ARnELl, K. M. (1992). Temporary suppression of visual processing in an RSVP task: An attentional blink? Journal of Experimental Psychology: Human Perception \& Performance, 18, 849-860.

Rensink, R. A., O'Regan, J. K., \& Clark, J. J. (1997). To see or not to see: The need for attention to perceive changes in scenes. Psychological Science, 8, 368-373.

Shelley-TREMBLAY,J., \& MACK, A. (1999). Metacontrast masking and attention. Psychological Science, 10, 508-515.

SimONS, D. J., \& LE VIN, D. T. (1998). Failure to detect changes to people during a real-world interaction. Psychonomic Bulletin \& Review, 5, 644-649.

SPENCER, T. J., \& Shuntich, R. (1970). Evidence for an interruption theory of backward masking. Journal of Experimental Psychology, 85, 198-203.

TAtA, M. S. (2002). Attend to it now or lose it forever: Selective attention, metacontrast masking, and object substitution. Perception \& Psychophysics, 64, 1028-1038.

TATA, M., \& GiAsCHI, D. (1999). Selective attention to the mask modulates object substitution masking. Investigative Ophthalmology \& Visual Science, 40, S806.

Treisman, A. M., \& Gelade, G. (1980). A feature integration theory of attention. Cognitive Psychology, 12, 97-136.

UTTAL, W. R. (1970). On the physiological basis of masking with dotted visual noise. Perception \& Psychophysics, 7, 321-327.

YANTIS, S., \& Johnson, D. N. (1990). Mechanisms of attentional priority. Journal of Experimental Psychology: Human Perception \& Performance, 16, 812-825.

YANTIS, S., \& JONIDES, J. (1984). Abrupt visual onsets and selective attention: Evidence from visual search. Journal of Experimental Psychology: Human Perception \& Performance, 10, 601-620.

(Manuscript received December 3, 2002; revision accepted for publication May 12, 2003.) 